\title{
Representation theory of $q$-rook monoid algebras
}

\author{
Rowena Paget
}

Received: 18 June 2005 / Accepted: 23 January 2006 / Published online: 11 July 2006

(C) Springer Science + Business Media, LLC 2006

\begin{abstract}
We show that, over an arbitrary field, $q$-rook monoid algebras are iterated inflations of Iwahori-Hecke algebras, and, in particular, are cellular. Furthermore we give an algebra decomposition which shows a $q$-rook monoid algebra is Morita equivalent to a direct sum of Iwahori-Hecke algebras. We state some of the consequences for the representation theory of $q$-rook monoid algebras.
\end{abstract}

Keywords Rook monoid · Cellular algebra $\cdot$ Iwahori-Hecke algebra $\cdot$ Representation

\section{Introduction}

The rook monoid is the monoid of all $n \times n$ matrices containing at most one entry equal to 1 in each row and column and zeros elsewhere, with the operation of matrix multiplication. It is also sometimes called the symmetric inverse semigroup. For any field $K$, we consider the rook monoid algebra $R_{n}$. This has been studied (mostly in the case where $K$ has characteristic zero) by, amongst others, Munn [13], Grood [6] and Solomon [14]. More generally, Solomon [15] defined a $q$-analogue of the rook monoid algebra and its representation theory was investigated by various authors in $[1,7,8]$.

We shall take the approach suggested by Henke in her review of [6] on MathSciNet, where it is stated that $R_{n}$ can be shown to be a cellular algebra using methods similar to those employed in [12]. Firstly we shall show that the $q$-rook monoid algebra is cellular, and in fact is an iterated inflation of Iwahori-Hecke algebras. Halverson and Ram (in [8]) already showed a connection between the $q$-rook monoid algebra and Hecke algebras - they showed that the $q$-rook monoid algebra is isomorphic to

Supported by EPSRC grant GR/S18151/01

R. Paget $(\bowtie)$

Mathematical Institute, 24-29 St. Giles, Oxford, OX1 3LB, England

e-mail: paget@maths.ox.ac.uk 
a quotient of the affine Hecke algebra of type $A$. We will find another connection. Our result will allow us to prove that the $q$-rook monoid algebra is actually Morita equivalent to a direct sum of Iwahori-Hecke algebras. This will allow us to reprove several results from the literature, as well as giving us some new results such as the block decomposition of these algebras.

Throughout, $K$ will denote an arbitrary field unless otherwise stated. All tensor products are taken over $K$.

\section{Background}

\subsection{Cellular algebras}

Cellular algebras were originally defined by Graham and Lehrer in [5], who proved that many familiar algebras are cellular, and also showed that cellular algebras possess many good properties. Let us recall the equivalent basis-free definition by König and $\mathrm{Xi}$ (from [10]). Let $K$ be a field, and $A$ be a finite dimensional $K$-algebra with an anti-involution $i$, that is a $K$-linear anti-automorphism $(i(a b)=i(b) i(a))$ of order 2 . A cell ideal of $A$ is a 2-sided ideal, $J$, satisfying:

(i) $i(J)=J$;

(ii) there exists $\Delta$, a left ideal of $A, \Delta \subset J$ and there is an $A-A$-bimodule isomorphism $\alpha: J \cong \Delta \otimes i(\Delta)$ such that the following diagram commutes:

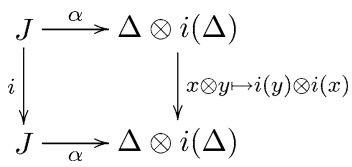

The algebra $A$ is cellular if there is a decomposition of $A$ as a vector space:

$$
A=J_{1}^{\prime} \oplus J_{2}^{\prime} \oplus \cdots \oplus J_{n}^{\prime}
$$

for some $n$, with $i\left(J_{j}^{\prime}\right)=J_{j}^{\prime}$, such that setting $J_{j}=J_{j}^{\prime} \oplus J_{j+1}^{\prime} \oplus \cdots \oplus J_{n}^{\prime}$ gives a chain of 2-sided ideals of $A$ :

$$
0=J_{n+1} \subset J_{n} \subset \cdots \subset J_{1}=A
$$

(each fixed by $i$ ), and, for each $j, J_{j}^{\prime}=J_{j} / J_{j+1}$ is a cell ideal of $A / J_{j+1}$ with respect to the anti-involution induced by $i$ on the quotient.

The left ideals of $A$ (that is left $A$-modules), $\Delta_{1}, \ldots, \Delta_{n}$ appearing in the definition are called the cell modules.

As an example of a cellular algebra, and for use later, we recall the Iwahori-Hecke algebra of the symmetric group. For $q \in K^{\times}$, the Hecke algebra of the symmetric group $\mathcal{H}_{n}(q)$ is defined to be the associative $K$-algebra with a 1 generated by $T_{1}, \ldots, T_{n-1}$ 祭 Springer 
subject to the relations:

$$
\begin{array}{ll}
T_{i}^{2}=q \cdot 1+(q-1) T_{i} & \text { for } 1 \leq i \leq n-1 \\
T_{i} T_{i+1} T_{i}=T_{i+1} T_{i} T_{i+1} & \text { for } 1 \leq i \leq n-2 \\
T_{i} T_{j}=T_{j} T_{i} & \text { when }|i-j|>1
\end{array}
$$

If we take $q=1$ we obtain the group algebra of the symmetric group $K \mathcal{S}_{n}$. As is wellknown, to each $w \in \mathcal{S}_{n}$ one can associate an element $T_{w} \in \mathcal{H}_{n}(q)$ to obtain a basis of $\mathcal{H}_{n}(q)$. Graham and Lehrer showed that $\mathcal{H}_{n}(q)$ (and, in particular, the symmetric group algebra) is a cellular algebra (see [5]), the anti-involution $i$ sending a generator $T_{j}$ to itself, and therefore sending a basis element $T_{w}$ to $T_{w^{-1}}$ for $w \in \mathcal{S}_{n}$.

We shall use the following lemma of Xi to demonstrate the cellularity of the $q$-rook monoid algebra. The lemma formalizes the method applied by König and Xi in [12] to show that Brauer algebras are cellular, ideas which first appeared in their article [11].

Lemma 1 ([16], 3.3). Let $A$ be a $K$-algebra with an anti-involution $i$. Suppose that the following hold:

(i) As a vector space,

$$
A=\bigoplus_{j=1}^{m} V_{j} \otimes V_{j} \otimes B_{j}
$$

and, for each $j, V_{j}$ is a vector space and $B_{j}$ is a cellular algebra with respect to anti-involution $\sigma_{j}$ and cell chain $0 \subset J_{s_{j}}^{(j)} \subset \cdots \subset J_{1}^{(j)}=B_{j}$.

(ii) The restriction of $i$ to $V_{j} \otimes V_{j} \otimes B_{j}$ is (for $v, v^{\prime} \in V_{j}, b \in B_{j}$ ):

$$
v \otimes v^{\prime} \otimes b \rightarrow v^{\prime} \otimes v \otimes \sigma_{j}(b)
$$

(iii) For each $j$, there is a bilinear form $\phi_{j}: V_{j} \otimes V_{j} \rightarrow B_{j}$ such that for all $v, v^{\prime} \in$ $V_{j}$ :

$$
\sigma_{j}\left(\phi_{j}\left(v^{\prime}, v\right)\right)=\phi_{j}\left(v, v^{\prime}\right)
$$

(iv) Putting $J_{t}=\bigoplus_{j=t}^{m} V_{j} \otimes V_{j} \otimes B_{j}$, the multiplication in $J_{j} / J_{j+1}$ is given by:

$$
\begin{aligned}
& \qquad\left(v_{1} \otimes v_{1}^{\prime} \otimes b_{1}\right)\left(v_{2} \otimes v_{2}^{\prime} \otimes b_{2}\right)=v_{1} \otimes v_{2}^{\prime} \otimes b_{1} \phi_{j}\left(v_{1}^{\prime}, v_{2}\right) b_{2} \quad \text { modulo } J_{j+1} \\
& \text { for } v_{1}, v_{1}^{\prime}, v_{2}, v_{2}^{\prime} \in V_{j}, b_{1}, b_{2} \in B_{j} .
\end{aligned}
$$

(v) For all $j, l$, we have that $V_{j} \otimes V_{j} \otimes J_{l}^{(j)}+J_{j+1}$ is an ideal in $A$.

Then under these conditions $A$ is a cellular algebra.

In this situation $A$ is called an iterated inflation of $B_{1}, B_{2}, \ldots, B_{m}$. For more details on inflations see Section 3 of [12]. 


\subsection{The $q$-rook monoid}

For $K$ a field of characteristic zero, $q \in K^{\times}$, Solomon defined the $q$-rook monoid algebra $\mathcal{I}_{n}(q)$ to be the associative $K$-algebra with identity 1 and generators $T_{1}, \ldots, T_{n-1}, N$ and defining relations:

$$
\begin{array}{ll}
T_{i}^{2}=q \cdot 1+(q-1) T_{i} & \text { for } 1 \leq i \leq n-1, \\
T_{i} T_{i+1} T_{i}=T_{i+1} T_{i} T_{i+1} & \text { for } 1 \leq i \leq n-2, \\
T_{i} T_{j}=T_{j} T_{i} & \text { when }|i-j|>1, \\
N^{i+1} T_{i}=q N^{i+1} & \text { for } 1 \leq i \leq n-1, \\
T_{i} N^{n-i+1}=q N^{n-i+1} & \text { for } 1 \leq i \leq n-1, \\
T_{i} N=N T_{i+1} & \text { for } 1 \leq i \leq n-2, \\
N T_{1} T_{2} \cdots T_{n-1} N=q^{n-1} N . &
\end{array}
$$

We will allow $K$ to be an arbitrary field in the above definition.

Halverson, in [7], defines $R_{n}(q)$ to be the associative $K$-algebra with identity generated by $T_{1}, \ldots, T_{n-1}$ and $P_{1}, \ldots, P_{n}$ subject to the relations:
(A1) $T_{i}^{2}=q \cdot 1+(q-1) T_{i} \quad$ for $1 \leq i \leq n-1$,
(A2) $T_{i} T_{i+1} T_{i}=T_{i+1} T_{i} T_{i+1} \quad$ for $1 \leq i \leq n-2$,
(A3) $T_{i} T_{j}=T_{j} T_{i}$
when $|i-j|>1$,
(A4) $T_{i} P_{j}=P_{j} T_{i}=q P_{j}$
for $1 \leq i<j \leq n$,
(A5) $T_{i} P_{j}=P_{j} T_{i}$
for $1 \leq j<i \leq n-1$,
(A6) $P_{i}^{2}=P_{i}$
for $1 \leq i \leq n$,
(A7) $P_{i+1}=P_{i} T_{i} P_{i}-(q-1) P_{i} \quad$ for $1 \leq i \leq n-1$.

Again Halverson is concerned only with the case where $K$ has characteristic zero, but we will allow $K$ to be any field in this definition.

Halverson proves [7, Corollary 2.2] that, over a field of characteristic zero, $\mathcal{I}_{n}(q) \cong$ $R_{n}(q)$. We will show that this result extends to the general case below. In Section 2 of [7], Halverson gave a basis of $R_{n}(q)$ defined over a field of characteristic zero, which we shall now recall. Let us denote $\{1,2, \ldots, n\}$ by $[n]$. For $A=\left\{a_{1}, \ldots, a_{k}\right\} \subseteq[n]$, with $a_{1}<a_{2}<\cdots<a_{k}$, we have $a_{j} \geq j$, so let:

$$
T_{A}=\left(T_{a_{1}-1} \cdots T_{2} T_{1}\right)\left(T_{a_{2}-1} \cdots T_{3} T_{2}\right) \cdots\left(T_{a_{k}-1} \cdots T_{k}\right),
$$

where the $j$-th factor is read as 1 if $a_{j}=j$. For $A, B \subseteq[n]$ with $|A|=|B|=k$, and $w \in \mathcal{S}_{\{k+1, \ldots, n\}}$, define:

$$
T_{(A, B, w)}=T_{A} P_{k} T_{w} T_{B}^{-1},
$$

where, when $k=0$, we interpret $P_{0}=1$ so $T_{(\emptyset, \emptyset, w)}=T_{w}$.

Write $\Omega_{k}=\left\{(A, B, w): A, B \subseteq[n],|A|=|B|=k, w \in \mathcal{S}_{\{k+1, \ldots, n\}}\right\}$ and $\Omega=$ $\bigcup_{k=0}^{n} \Omega_{k}$. Halverson proves that, when the characteristic of $K$ is zero, the followSpringer 
ing set is a basis of $R_{n}(q)$ :

$$
\left\{T_{(A, B, w)}:(A, B, w) \in \Omega\right\}
$$

Proposition 2 ([7, Corollary 2.2] over a field of characteristic zero). Over an arbitraryfield $\mathcal{I}_{n}(q) \cong R_{n}(q)$. Moreover $\left\{T_{(A, B, w)}:(A, B, w) \in \Omega\right\}$ forms a basis of $R_{n}(q)$.

Proof: We shall see that Halverson's proof of this in [7] generalises to this situation. Halverson proves that the set in (1) spans $R_{n}(q)$ in [7, Theorem 2.1] and this proof is readily seen to hold for an arbitrary field. So the dimension of $R_{n}(q)$ is at most $|\Omega|$. He shows that one can define a surjection from $R_{n}(q)$ to $\mathcal{I}_{n}(q)$ (see [7, Lemma 1.4]) and this is valid over any field. Thus $\operatorname{dim}_{K}\left(\mathcal{I}_{n}(q)\right) \leq \operatorname{dim}_{K}\left(R_{n}(q)\right) \leq|\Omega|$. To get equality, Halverson appeals to Solomon's proof that the dimension of $\mathcal{I}_{n}(q)$ is at least $|\Omega|$ (see [15, Remark 2.28]). The argument here is that if there is an algebra $\mathcal{I}^{\prime}$ of dimension $|\Omega|$ which satisfies the defining relations of $\mathcal{I}_{n}(q)$, then $\mathcal{I}^{\prime}$ is a homomorphic image of $\mathcal{I}_{n}(q)$ and $\operatorname{so} \operatorname{dim}_{K}\left(\mathcal{I}_{n}(q)\right) \geq \operatorname{dim}_{K}\left(\mathcal{I}^{\prime}\right)=|\Omega|$. Solomon defines such an algebra ([15], $2.33,2.34)$ and shows that it can be defined for any field $K$. This therefore means that Halverson's proof that $\mathcal{I}_{n}(q) \cong R_{n}(q)$ is valid for the algebras defined over an arbitrary field, and also shows that (1) is a basis of $R_{n}(q)$.

From now on we shall use Halverson's presentation, and write $R_{n}(q)$ for the $q$-rook monoid algebra.

The $q$-rook monoid algebra is indeed a $q$-analogue of the rook monoid, as one sees by setting $q=1$ where we obtain the rook monoid algebra $R_{n}$. The generators $T_{i}$ become the permutation matrices associated to the transpositions $(i, i+1)$, and $P_{i}$ becomes $E_{i+1, i+1}+\cdots+E_{n, n}$ (where $E_{i, j}$ denotes the elementary matrix with a 1 in position $i, j$ ). If we set $q=1$ in Solomon's presentation then the generator $N$ should be identified with $E_{1,2}+E_{2,3}+\cdots+E_{n-1, n}$.

Let us record a few obvious statements which can be deduced from the relations:

$$
\begin{aligned}
T_{i}^{-1} & =\left(q^{-1}-1\right) \cdot 1+q^{-1} T_{i}, \\
P_{i} P_{j} & =P_{j} \quad \text { if } i \leq j, \\
P_{i} T_{i}^{-1} P_{i} & =q^{-1} P_{i+1} .
\end{aligned}
$$

The subalgebra of $R_{n}(q)$ generated by $T_{1}, T_{2}, \ldots, T_{n-1}$ is isomorphic to $\mathcal{H}_{n}(q)$. So for $w \in \mathcal{S}_{n}$ one can associate an element $T_{w} \in R_{n}(q)$ in the usual way.

\section{A new basis}

\subsection{The anti-involution}

As we saw above, part of the definition of a cellular algebra is the existence of an antiinvolution on the algebra. We define an anti-involution, that is an anti-automorphism 
$i$ satisfying $i^{2}=\mathrm{id}$, on $R_{n}(q)$ by extending:

$$
\begin{aligned}
i: R_{n}(q) & \rightarrow R_{n}(q) \\
i\left(T_{j}\right) & =T_{j} \quad \text { for } 1 \leq j \leq n-1 \\
i\left(P_{1}\right) & =P_{1} .
\end{aligned}
$$

From Eq. (2) we see $i\left(T_{j}^{-1}\right)=T_{j}^{-1}$, and thus from Eq. (4) we obtain $i\left(P_{j}\right)=P_{j}$ for $1 \leq j \leq n$.

\subsection{A chain of ideals}

For $1 \leq k \leq n$, let us write $J_{k}$ to denote the 2-sided ideal of $R_{n}(q)$ generated by $P_{k}$, (and for convenience let us write $J_{0}=R_{n}(q)$ and $J_{n+1}=0$ ). Then this gives a chain of 2-sided ideals:

$$
0=J_{n+1} \subset J_{n} \subset J_{n-1} \subset \cdots \subset J_{1} \subset J_{0}=R_{n}(q) .
$$

Solomon also uses these ideals (see [15], (1.10)), where they are written $\Theta_{k}$.

\subsection{A new basis}

We shall use a slightly different basis of $R_{n}(q)$ than that defined by Halverson, and recalled above. For $(A, B, w) \in \Omega_{k}, k=0,1, \ldots, n$, define:

$$
\tilde{T}_{(A, B, w)}:=i\left(T_{A}^{-1}\right) P_{k} T_{w} T_{B}^{-1} .
$$

Proposition 3. The set $\left\{\tilde{T}_{(A, B, w)}:(A, B, w) \in \Omega\right\}$ is a basis of $R_{n}(q)$.

Proof: We shall see that Halverson's basis elements $T_{(A, B, w)}$ can be expressed as linear combinations of the elements $\tilde{T}_{\left(C, D, w^{\prime}\right)}$. We use induction on the length of $T_{A}$. If the length is zero then $A=\{1,2, \ldots, k\}$ and $T_{(A, B, w)}=\tilde{T}_{(A, B, w)}$. Otherwise write $T_{(A, B, w)}$ as $T_{j} T_{\left(A^{\prime}, B, w\right)}$, where $j=a_{i}-1$ if $\left\{a_{1}, \ldots, a_{i-1}\right\}=\{1, \ldots, i-1\}$ but $a_{i} \neq i$, and $A^{\prime}=\left\{1, \ldots, a_{i-1}, a_{i}-1, a_{i+1}, \ldots, a_{k}\right\}$. The inductive hypothesis tells us we can express $T_{\left(A^{\prime}, B, w\right)}$ in the required form so it remains to check that $T_{j} \tilde{T}_{(C, D, u)}$, or equivalently (using the expression for inverses)

$T_{j}^{-1} \tilde{T}_{(C, D, u)}$ can be written as a linear combination of elements $\tilde{T}_{\left(C, D, w^{\prime}\right)}$. To do this we refer to the proof of Theorem 2.1 in [7]. Here Halverson shows that $P_{k} T_{C}^{-1} T_{j}^{-1}$ can be written as a linear combination of elements $T_{u^{\prime}} P_{k} T_{C}^{-1}$ (for $u^{\prime} \in \mathcal{S}_{\{k+1, \ldots, n\}}$ ). But then applying the anti-involution $i$ shows that $T_{j}^{-1} i\left(T_{C}^{-1}\right) P_{k}$ is a linear combination of elements $i\left(T_{C}^{-1}\right) P_{k} T_{u^{\prime-1}}$. So $T_{j}^{-1} \tilde{T}_{(C, D, u)}$ can be written as a sum of elements $i\left(T_{C}^{-1}\right) P_{k}\left(T_{u^{\prime-1}} T_{u}\right) T_{D}^{-1}$, which can be written in the required form. 
Clearly the images of the basis elements $\tilde{T}_{(A, B, w)}$ for $(A, B, w) \in \Omega_{k}$ form a basis of $J_{k} / J_{k+1}$, for $0 \leq k \leq n$. Observe also that $i\left(\tilde{T}_{(A, B, w)}\right)=\tilde{T}_{\left(B, A, w^{-1}\right)}$.

\section{Basis calculations}

Our aim is to show that $R_{n}(q)$ is an iterated inflation of Hecke algebras, coming from the chain of ideals (5). To do this we shall need to understand how to multiply elements within a layer $J_{k} / J_{k+1}$, where $0 \leq k \leq n$. Suppose $(A, B, w),\left(C, D, w^{\prime}\right) \in \Omega_{k}$. Then (A5) implies that $P_{k} T_{w}=T_{w} P_{k}$, hence:

$$
\begin{aligned}
\tilde{T}_{(A, B, w)} \tilde{T}_{\left(C, D, w^{\prime}\right)} & =i\left(T_{A}^{-1}\right) P_{k} T_{w} T_{B}^{-1} i\left(T_{C}^{-1}\right) P_{k} T_{w^{\prime}} T_{D}^{-1} \\
& =i\left(T_{A}^{-1}\right) T_{w} X_{B, C} T_{w^{\prime}} T_{D}^{-1}
\end{aligned}
$$

where

$$
X_{B, C}=P_{k} T_{B}^{-1} i\left(T_{C}^{-1}\right) P_{k}
$$

Suppose that $B=\left\{b_{1}, \ldots, b_{k}\right\}, C=\left\{c_{1} \ldots, c_{k}\right\}$ where $b_{1}<b_{2}<\cdots<b_{k}$ and $c_{1}<$ $c_{2}<\cdots<c_{k}$. Observe that by the relation (A5) along with Eq. (3), we can write $X_{B, C}$ in the following way:

$$
\begin{aligned}
X_{B, C}= & P_{k}\left(T_{k}^{-1} T_{k+1}^{-1} \cdots T_{b_{k}-1}^{-1}\right) P_{k-1}\left(T_{k-1}^{-1} T_{k}^{-1} \cdots T_{b_{k-1}-1}^{-1}\right) P_{k-2} \cdots \\
& \cdots P_{1}\left(T_{1}^{-1} T_{2}^{-1} \cdots T_{b_{1}-1}^{-1}\right)\left(T_{c_{1}-1}^{-1} \cdots T_{2}^{-1} T_{1}^{-1}\right) P_{1} \cdots \\
& \cdots P_{k-2}\left(T_{c_{k-1}-1}^{-1} \cdots T_{k}^{-1} T_{k-1}^{-1}\right) P_{k-1}\left(T_{c_{k}-1}^{-1} \cdots T_{k+1}^{-1} T_{k}^{-1}\right) P_{k} .
\end{aligned}
$$

Lemma 4. Suppose $b, c>i$ and $b \neq c$. Then:

$$
\begin{aligned}
P_{i} & T_{i}^{-1} T_{i+1}^{-1} \cdots T_{b-1}^{-1} T_{c-1}^{-1} \cdots T_{i+1}^{-1} T_{i}^{-1} P_{i} \\
& =\left\{\begin{array}{l}
q^{-1} T_{c-1}^{-1} \cdots T_{i+1}^{-1} P_{i+1} T_{i+1}^{-1} \cdots T_{b}^{-1} \text { if } b<c \\
q^{-1} T_{c}^{-1} \cdots T_{i+1}^{-1} P_{i+1} T_{i+1}^{-1} \cdots T_{b-1}^{-1} \text { if } b>c .
\end{array}\right.
\end{aligned}
$$

Proof: Consider the case $b<c$. By (A3) and (A5),

$$
\begin{aligned}
& P_{i} T_{i}^{-1} T_{i+1}^{-1} \cdots T_{b-1}^{-1} T_{c-1}^{-1} \cdots T_{b+1}^{-1} T_{b}^{-1} T_{b-1}^{-1} \cdots T_{i+1}^{-1} T_{i}^{-1} P_{i} \\
& \quad=T_{c-1}^{-1} \cdots T_{b+1}^{-1} P_{i} T_{i}^{-1} T_{i+1}^{-1} \cdots T_{b-1}^{-1} T_{b}^{-1} T_{b-1}^{-1} \cdots T_{i+1}^{-1} T_{i}^{-1} P_{i} .
\end{aligned}
$$


Now use (A2) to write $T_{b-1}^{-1} T_{b}^{-1} T_{b-1}^{-1}=T_{b}^{-1} T_{b-1}^{-1} T_{b}^{-1}$, and then apply (A3) and (A5) again to get equality with:

$$
T_{c-1}^{-1} \cdots T_{b+1}^{-1} T_{b}^{-1} P_{i} T_{i}^{-1} T_{i+1}^{-1} \cdots T_{b-2}^{-1} T_{b-1}^{-1} T_{b-2}^{-1} \cdots T_{i+1}^{-1} T_{i}^{-1} P_{i} T_{b}^{-1} .
$$

Repeat this argument until we reach:

$$
T_{c-1}^{-1} \cdots T_{i+1}^{-1} P_{i} T_{i}^{-1} P_{i} T_{i+1}^{-1} \cdots T_{b}^{-1}=q^{-1} T_{c-1}^{-1} \cdots T_{i+1}^{-1} P_{i+1} T_{i+1}^{-1} \cdots T_{b}^{-1}
$$

by Eq. (4). The other case is analogous.

Lemma 5. Suppose $b_{i}<\cdots<b_{k}$ and $c_{i}<\cdots<c_{k}$ with $b_{i} \neq c_{i}$. Then:

$$
P_{k}\left(T_{k}^{-1} \cdots T_{b_{k}-1}^{-1}\right) P_{k-1} \cdots P_{i}\left(T_{i}^{-1} \cdots T_{b_{i}-1}^{-1}\right)\left(T_{c_{i}-1}^{-1} \cdots T_{i}^{-1}\right) P_{i} \cdots P_{k-1}\left(T_{c_{k}-1}^{-1} \cdots T_{k}^{-1}\right) P_{k}
$$

lies in $J_{k+1}$.

Proof: Consider the case $c_{i}<b_{i}$ (as the other case is analogous). Use Lemma 4 to replace

$$
P_{i} T_{i}^{-1} \cdots T_{b_{i}-1}^{-1} T_{c_{i}-1}^{-1} \cdots T_{i}^{-1} P_{i}
$$

by $q^{-1} T_{c_{i}}^{-1} \cdots T_{i+1}^{-1} P_{i+1} T_{i+1}^{-1} \cdots T_{b_{i}-1}^{-1}$. Now consider the subexpression

$$
P_{i+1} T_{i+1}^{-1} \cdots T_{b_{i+1}-1}^{-1} T_{c_{i}}^{-1} \cdots T_{i+1}^{-1} P_{i+1} .
$$

As $c_{i}<b_{i}<b_{i+1}$, we have $c_{i}<b_{i+1}-1$, so we can use Lemma 4 again and replace the subexpression by $q^{-1} T_{c_{i}+1}^{-1} \cdots T_{i+2}^{-1} P_{i+2} T_{i+2}^{-1} \cdots T_{b_{i+1}-1}^{-1}$. Keep repeating this step until we have shown that:

$$
\begin{aligned}
& P_{k}\left(T_{k}^{-1} \cdots T_{b_{k}-1}^{-1}\right) P_{k-1} \cdots P_{i}\left(T_{i}^{-1} \cdots T_{b_{i}-1}^{-1}\right)\left(T_{c_{i}-1}^{-1} \cdots T_{i}^{-1}\right) P_{i} \cdots P_{k-1}\left(T_{c_{k}-1}^{-1} \cdots T_{k}^{-1}\right) P_{k} \\
& \quad=T_{c_{i}+k-i}^{-1} \cdots T_{k+1}^{-1} P_{k+1} u
\end{aligned}
$$

for some element $u \in R_{n}(q)$.

Lemma 6. For $a \geq 0$,

$$
\begin{aligned}
& P_{k} T_{k}^{-1} T_{k+1}^{-1} \cdots T_{k+a}^{-1} T_{k+a}^{-1} \cdots T_{k+1}^{-1} T_{k}^{-1} P_{k} \\
& \quad=q^{-(a+1)} P_{k}+q^{-(a+2)}(1-q) P_{k+1}+(1-q) \sum_{j=1}^{a} q^{-a+j-2} T_{k+j}^{-1} \cdots T_{k+1}^{-1} P_{k+1} T_{k+1}^{-1} \cdots T_{k+j}^{-1} .
\end{aligned}
$$


Proof: When $a=0$ we have

$$
P_{k}\left(T_{k}^{-1}\right)^{2} P_{k}=P_{k}\left(q^{-1} \cdot 1+(1-q) q^{-1} T_{k}^{-1}\right) P_{k}=q^{-1} P_{k}+q^{-2}(1-q) P_{k+1} .
$$

For $a \geq 1$, substitute $\left(T_{k+a+1}^{-1}\right)^{2}=q^{-1} \cdot 1+(1-q) q^{-1} T_{k+a+1}^{-1}$ into the following: $P_{k} T_{k}^{-1} T_{k+1}^{-1} \cdots T_{k+a}^{-1}\left(T_{k+a+1}^{-1}\right)^{2} T_{k+a}^{-1} \cdots T_{k+1}^{-1} T_{k}^{-1} P_{k}$. Use the inductive hypothesis for the first term, and use Lemma 4 for the second. The result follows by induction.

Lemma 7. Let $B=\left\{b_{1}, b_{2}, \ldots, b_{k}\right\}$ with $b_{1}<\cdots<b_{k}$. Then $X_{B, B}$ is equal to

$$
q^{-\left(b_{1}-1\right)-\cdots-\left(b_{k}-k\right)} P_{k}
$$

plus terms of the form

$$
T_{j}^{-1} T_{j-1}^{-1} \cdots T_{k+1}^{-1} P_{k+1} u_{j} P_{k+1} T_{k+1}^{-1} \cdots T_{j-1}^{-1} T_{j}^{-1},
$$

for $k \leq j \leq b_{k}-1$, where each $u_{j} \in R_{n}(q)$ (and we read this as $P_{k+1} u_{k} P_{k+1}$ for $j=k)$.

Proof: We prove this by induction. When $k=1$ this has the required form by Lemma 6 . For $k>1$, write $B^{\prime}=\left\{b_{1}, \ldots, b_{k-1}\right\}$. Then

$$
X_{B, B}=P_{k}\left(T_{k}^{-1} \cdots T_{b_{k}-1}^{-1}\right) X_{B^{\prime}, B^{\prime}}\left(T_{b_{k}-1}^{-1} \cdots T_{k}^{-1}\right) P_{k}
$$

which, by the inductive hypothesis, equals

$$
q^{-\left(b_{1}-1\right)-\ldots-\left(b_{k-1}-k+1\right)} P_{k}\left(T_{k}^{-1} \cdots T_{b_{k}-1}^{-1}\right)\left(T_{b_{k}-1}^{-1} \cdots T_{k}^{-1}\right) P_{k},
$$

plus terms of the form:

$$
P_{k}\left(T_{k}^{-1} \cdots T_{b_{k}-1}^{-1}\right) T_{j}^{-1} \cdots T_{k}^{-1} P_{k} u_{j} P_{k} T_{k}^{-1} \cdots T_{j}^{-1}\left(T_{b_{k}-1}^{-1} \cdots T_{k}^{-1}\right) P_{k}
$$

for $k-1 \leq j \leq b_{k-1}-1<b_{k}-1$. Lemma 6 shows that the first term equals $q^{-\left(b_{1}-1\right)-\cdots-\left(b_{k}-\bar{k}\right)} P_{k}$ plus terms of the required form. The other terms above can be written as:

$$
P_{k}\left(T_{k}^{-1} \cdots T_{j}^{-1} T_{j+1}^{-1} T_{j}^{-1} \cdots T_{k}^{-1} P_{k} u_{j}^{\prime} P_{k} T_{k}^{-1} \cdots T_{j}^{-1} T_{j+1}^{-1} T_{j}^{-1} \cdots T_{k}^{-1}\right) P_{k}
$$

for some $u_{j}^{\prime} \in R_{n}(q)$. Applying Lemma 4, this equals

$$
q^{-2} T_{j+1}^{-1} T_{j}^{-1} \cdots T_{k+1}^{-1} P_{k+1} T_{k+1}^{-1} \cdots T_{j+1}^{-1} u_{j}^{\prime} T_{j+1}^{-1} T_{j}^{-1} \cdots T_{k+1}^{-1} P_{k+1} T_{k+1}^{-1} \cdots T_{j+1}^{-1} .
$$

Writing this as $T_{j+1}^{-1} T_{j}^{-1} \cdots T_{k+1}^{-1} P_{k+1} u_{j}^{\prime \prime} P_{k+1} T_{k+1}^{-1} \cdots T_{j+1}^{-1}$, we see it has the required form. 
Proposition 8. Suppose $|B|=|C|=k$. Then modulo $J_{k+1}$,

$$
X_{B, C}= \begin{cases}q^{\alpha_{B}} P_{k} & \text { if } B=C \\ 0 & \text { if } B \neq C .\end{cases}
$$

where, if $B=\left\{b_{1}, b_{2}, \ldots, b_{k}\right\}, \alpha_{B}=-\sum_{i=1}^{k} b_{i}+\frac{k(k+1)}{2}$.

Proof: Lemma 7 deals with the case $B=C$. Now assume $B \neq C$. If $b_{1} \neq c_{1}$ then $X_{B, C} \in J_{k+1}$ by Lemma 5. So assume $B^{\prime}:=\left\{b_{1}, \ldots, b_{i-1}\right\}=\left\{c_{1}, \ldots, c_{i-1}\right\}$ but $b_{i} \neq$ $c_{i}$. Then

$$
\begin{aligned}
X_{B, C}= & P_{k}\left(T_{k}^{-1} \cdots T_{b_{k}-1}^{-1}\right) P_{k-1} \cdots P_{i}\left(T_{i}^{-1} \cdots T_{b_{i}-1}^{-1}\right) X_{B^{\prime}, B^{\prime}} \\
& \left(T_{c_{i}-1}^{-1} \cdots T_{i}^{-1}\right) P_{i} \cdots P_{k-1}\left(T_{c_{k}-1}^{-1} \cdots T_{k}^{-1}\right) P_{k}
\end{aligned}
$$

Use Lemma 7 to express $X_{B^{\prime}, B^{\prime}}$, and substitute into the above. We obtain a term of a unit times:

$$
P_{k}\left(T_{k}^{-1} \cdots T_{b_{k}-1}^{-1}\right) P_{k-1} \cdots P_{i}\left(T_{i}^{-1} \cdots T_{b_{i}-1}^{-1}\right)\left(T_{c_{i}-1}^{-1} \cdots T_{i}^{-1}\right) P_{i} \cdots P_{k-1}\left(T_{c_{k}-1}^{-1} \cdots T_{k}^{-1}\right) P_{k}
$$

which lies in $J_{k+1}$ by Lemma 5, and also terms of the form:

$$
P_{k}\left(T_{k}^{-1} \cdots T_{b_{k}-1}^{-1}\right) P_{k-1} \cdots P_{i}\left(T_{i}^{-1} \cdots T_{b_{i}-1}^{-1}\right)\left(T_{j}^{-1} \cdots T_{i}^{-1}\right) P_{i} w
$$

(where $i-1 \leq j<b_{i-1}-1<b_{i}-1$ and $w \in R_{n}(q)$ ). Now repeatedly apply Lemma 4 , firstly replacing $P_{i}\left(T_{i}^{-1} \cdots T_{b_{i}-1}^{-1}\right)\left(T_{j}^{-1} \cdots T_{i}^{-1}\right) P_{i} w$ by $T_{j+1}^{-1} \cdots T_{i+1}^{-1}$ $P_{i+1} w^{\prime}$, for some $w^{\prime} \in R_{n}(q)$, until we arrive at $P_{k}\left(T_{k}^{-1} \cdots T_{b_{k}-1}^{-1}\right)\left(T_{j^{\prime}}^{-1} T_{j^{\prime}+1}^{-1} \cdots\right.$ $\left.T_{k}^{-1}\right) P_{k} \bar{w}$ for some $j^{\prime}<b_{k}-1$ and some $\bar{w} \in R_{n}(q)$. By Lemma 4 , this lies in $J_{k+1}$.

\section{Main results}

Our calculations in the previous section have shown us how to multiply within a layer.

Theorem 9. Let $(A, B, w),\left(C, D, w^{\prime}\right) \in \Omega_{k}$. Then, if $B \neq C$,

$$
\tilde{T}_{(A, B, w)} \tilde{T}_{\left(C, D, w^{\prime}\right)}=0\left(\bmod J_{k+1}\right)
$$

and, if $B=C$,

$$
\tilde{T}_{(A, B, w)} \tilde{T}_{\left(C, D, w^{\prime}\right)}=q^{\alpha_{B}} \sum_{u} \gamma_{u} \tilde{T}_{(A, D, u)}\left(\bmod J_{k+1}\right)
$$


where $T_{w} T_{w^{\prime}}=\sum_{u \in \mathcal{S}_{\{k+1, \ldots, n\}}} \gamma_{u} T_{u}$ is the multiplication in $\mathcal{H}_{\{k+1, \ldots, n\}}(q)$, and, if $B=$ $\left\{b_{1}, \ldots, b_{k}\right\}$, then $\alpha_{B}=-\sum_{i=1}^{k} b_{i}+\frac{k(k+1)}{2}$.

We can translate this into the language of inflations as follows. For $k=0,1, \ldots, n$, let $V_{k}$ denote a $K$-vector space with basis labelled by subsets of $[n]$ of size $k$, that is $\left\{v_{A}: A \subseteq[n],|A|=k\right\}$. Now define a bilinear form $\phi: V_{k} \times V_{k} \rightarrow K$ by extending:

$$
\phi\left(v_{B}, v_{C}\right)= \begin{cases}q^{\alpha_{B}} & \text { if } B=C, \\ 0 & \text { if } B \neq C .\end{cases}
$$

where $\alpha_{B}$ is the coefficient appearing in Theorem 9 above

\section{Corollary 10.}

$$
J_{k} / J_{k+1} \cong V_{k} \otimes V_{k} \otimes \mathcal{H}_{n-k}(q),
$$

where the multiplication is given by:

$$
\left(v_{A} \otimes v_{B} \otimes T_{w}\right)\left(v_{C} \otimes v_{D} \otimes T_{w^{\prime}}\right)=v_{A} \otimes v_{D} \otimes\left(T_{w} \phi\left(v_{B}, v_{C}\right) T_{w^{\prime}}\right)
$$

The cellularity of the $q$-rook monoid algebra will now follow from Xi's statement (Lemma 1) once we verify that condition (v) of that lemma holds. This is done next.

Lemma 11. If I is an ideal of $\mathcal{H}_{\{k+1, \ldots, n\}}(q)$ then $\tilde{I}=V_{k} \otimes V_{k} \otimes I+J_{k+1}$ is an ideal of $R_{n}(q)$.

Proof: Take $i\left(T_{A}^{-1}\right) P_{k} y T_{B}^{-1}=i\left(T_{A}^{-1}\right) P_{k} y P_{k} T_{B}^{-1}$ where $|A|=|B|=k$ and $y \in I$ and consider right-multiplication by a basis element, $\tilde{T}_{(C, D, w)}$ (and the argument for leftmultiplication is identical). By noting that $P_{k} T_{B}^{-1} \tilde{T}_{(C, D, w)} \in J_{k}$, it is sufficient to consider $i\left(T_{A}^{-1}\right) P_{k} y \tilde{T}_{\left(E, F, w^{\prime}\right)}=i\left(T_{A}^{-1}\right) y P_{k} \tilde{T}_{\left(E, F, w^{\prime}\right)}$ for $\left(E, F, w^{\prime}\right) \in \Omega_{k}$, whence we can apply Proposition 8 to see that the product is in $\tilde{I}$.

Corollary 12. The q-rook monoid algebra $R_{n}(q)$ is an iterated inflation of the Hecke algebras: $\mathcal{H}_{n}(q), \mathcal{H}_{n-1}(q), \ldots, \mathcal{H}_{1}(q), \mathcal{H}_{0}(q)=K$. Thus $R_{n}(q)$ is a cellular algebra.

The rook monoid algebra (the case $q=1$ ) was already known to be a cellular algebra by work of East in [4]. Corollary 12 tells us that, as a vector space:

$$
R_{n}(q) \cong \bigoplus_{k=0}^{n}\left(V_{k} \otimes V_{k} \otimes \mathcal{H}_{n-k}(q)\right)
$$

where $\mathcal{H}_{0}(q)$ is read as $K$. In fact this is more than just a vector space decomposition. To see this we use the following lemma of König and Xi. 
Lemma 13 ([12], Lemma 7.1). If $A$ is a ring and $J$ a 2 -sided ideal in $A$ then $J \subseteq A$ induces a ring decomposition $A \cong J \oplus(A / J)$ if and only if $J$ has a unit element.

We briefly recall the proof. One direction is trivial. For the other, assume there is some $e \in J$ such that $e x=x e=x$ for all $x \in J$. Then $J=e J e \subseteq e A e \subseteq J$, so $J=$ $e A e$. Then $\left(1_{A}-e\right) A e \subseteq\left(1_{A}-e\right) J=\left(1_{A}-e\right) e J=0$ and similarly $e A\left(1_{A}-e\right)=$ 0 . Hence $A=e A e \oplus\left(1_{A}-e\right) A\left(1_{A}-e\right)$.

Theorem 14. As an algebra,

$$
R_{n}(q) \cong \bigoplus_{k=0}^{n}\left(V_{k} \otimes V_{k} \otimes \mathcal{H}_{n-k}(q)\right)
$$

where $\mathcal{H}_{0}(q)$ is read as $K$.

Proof: By Lemma 13, it is sufficient to find a unit element in each layer. In $J_{n}$ we define $e_{n}=P_{n}$, which is certainly a unit in $J_{n}$. Now in $J_{k} / J_{k+1} \cong V_{k} \otimes V_{k} \otimes \mathcal{H}_{n-k}(q)$, define $e_{k}=\sum_{A} \frac{1}{\phi\left(v_{A}, v_{A}\right)} \tilde{T}_{(A, A, 1)}$. The images of $\left\{\tilde{T}_{(B, C, w)}:(B, C, w) \in \Omega_{k}\right\}$ form a basis of $J_{k} / J_{k+1}$, and:

$$
\begin{aligned}
\tilde{T}_{(B, C, w)} e_{k} & =\sum_{A} \frac{1}{\phi\left(v_{A}, v_{A}\right)} \tilde{T}_{(B, C, w)} \tilde{T}_{(A, A, 1)} \\
& =\frac{1}{\phi\left(v_{C}, v_{C}\right)} \tilde{T}_{(B, C, w)} \tilde{T}_{(C, C, 1)}\left(\bmod J_{k+1}\right) \\
& =\tilde{T}_{(B, C, w)}\left(\bmod J_{k+1}\right)
\end{aligned}
$$

by Corollary 10. Similarly $e_{k} \tilde{T}_{(B, C, w)}=\tilde{T}_{(B, C, w)}\left(\bmod J_{k+1}\right)$, and the image of $e_{k}$ in $J_{k} / J_{k+1}$ is the required unit.

Solomon proves this for the rook monoid (that is $q=1$ case) over a field of characteristic zero, in [14, Corollary 2.19], and it is originally a result of Munn. Although the assumption on the characteristic is required for later results in Solomon's paper, it seems not to be needed for the arguments leading up to Corollary 2.19, so his proof would also apply to a field of non-zero characteristic when $q=1$.

We have the following corollary.

Corollary 15. Over any field $R_{n}(q)$ is Morita equivalent to

$$
\mathcal{H}_{n}(q) \oplus \mathcal{H}_{n-1}(q) \oplus \cdots \oplus \mathcal{H}_{1}(q) \oplus K
$$

Proof: This follows from the theory of inflations in [12]. The bilinear form $\phi: V_{k} \times$ $V_{k} \rightarrow K$ is non-singular, and thus (by [12], 3.4) we have a Morita equivalence between $V_{k} \otimes V_{k} \otimes \mathcal{H}_{n-k}(q)$ and $\mathcal{H}_{n-k}(q)$.

Springer 
This means that the representation theory of the $q$-rook monoid algebra is the same as that of the above direct sum of Hecke algebras. This allows us to answer many representation theoretic questions about $R_{n}(q)$.

- The cell modules of $R_{n}(q)$ are, as vector spaces, isomorphic to $S^{\lambda} \otimes V_{l}$ for $0 \leq l \leq n$ and $\lambda$ a partition of $n-l$, where $S^{\lambda}$ denotes the usual Specht module for $\mathcal{H}_{n-1}(q)$ (as defined in Section 4 of [2]). If $R_{n}(q)$ is semisimple then these form a complete set of non-isomorphic simple $R_{n}(q)$-modules. However, in general they need not be simple. Let $e$ be the least positive integer such that

$$
1+q+q^{2}+\cdots+q^{e-1}=0
$$

if such an integer exists, and put $e=\infty$ otherwise. Then the simple $R_{n}(q)$ modules are labelled by the $e$-regular partitions $\lambda$ of $n-l, 0 \leq l \leq n$. As vector spaces these are isomorphic to $D^{\lambda} \otimes V_{l}$ where $D^{\lambda}$ is a simple $\mathcal{H}_{n-l}(q)$-module (as in [2] Sections 4 and 6.1). In particular this shows that the dimension of the simple $R_{n}(q)$-module labelled by $\lambda$ equals $\left(\begin{array}{l}n \\ l\end{array}\right) \operatorname{dim}_{K}\left(D^{\lambda}\right)$.

- The block decomposition of $R_{n}(q)$ is given by the so-called Nakayama Conjecture (see Section 4 of [3]). Associated to a partition $\lambda$ is its $e$-core, obtained by deleting all rim $e$-hooks from the Young diagram of $\lambda$ (see [9], Section 2.7). If $\lambda$ is a partition with $e$-core $\kappa$ then $|\lambda|=|\kappa|+e w$ for some $w$ which is called the $e$-weight of $\lambda$. Two cell modules for $R_{n}(q), S^{\lambda} \otimes V_{l}$ and $S^{\mu} \otimes V_{k}$ lie in the same block if and only if $\lambda$ and $\mu$ have the same $e$-cores and $e$-weights.

- $R_{n}(q)$ is a semisimple algebra if and only if $n<e$. This was proved by Halverson and Ram in the case when $K=\mathbb{C}$ (see [8], 2.21). A block of $R_{n}(q)$ is simple if and only if its $e$-weight equals zero. A block of $R_{n}(q)$ is of finite type if and only if its $e$-weight is at most 1 .

- The decomposition matrix of a cellular algebra records the multiplicity of each simple module in each cell module. The decomposition matrix of $R_{n}(q)$ is just the block diagonal matrix containing the decomposition matrices of the Hecke algebras $\mathcal{H}_{n}(q), \mathcal{H}_{n-1}(q), \ldots, \mathcal{H}_{1}(q), K$.

- In particular, the rook monoid algebra $R_{n}$ (that is the $q=1$ case) is Morita equivalent to $K \mathcal{S}_{n} \oplus K \mathcal{S}_{n-1} \oplus \cdots \oplus K \mathcal{S}_{1} \oplus K$. As noted in Henke's review of [6], the Morita equivalence of Corollary 15 in the case when $q=1$ sends Specht modules for symmetric groups to the Specht modules constructed by Grood-these are just the cell modules of $R_{n}, S^{\lambda} \otimes V_{l}$ where $S^{\lambda}$ is a Specht module of $K \mathcal{S}_{n-l}$ (as defined in Section 7.1 of [9]) for $l \in\{0,1, \ldots, n\}$.

Acknowledgments I would like to thank Robert Hartmann for his initial computer calculations about the rook monoid, and also Steffen König and Karin Erdmann for many helpful discussions.

\section{References}

1. M. Dieng, T. Halverson, and V. Poladian, "Character formulas for $q$-Rook monoid algebras," J. Algebraic Combin. 17 (2003), 99-123.

2. R. Dipper, and G. James, "Representations of Hecke algebras of general linear groups," Proc. London Math. Soc. 52(3) (1986), 20-52. 
3. R. Dipper, G. James, "Blocks and idempotents of Hecke algebras of general linear groups," Proc. London Math. Soc. 54(3) (1987), 57-82.

4. J. East, "Cellular algebras and inverse semigroups," preprint.

5. J. Graham and G. Lehrer, "Cellular algebras," Inv. Math. 123 (1996), 1-34.

6. C. Grood, "A Specht module analog for the rook monoid," Electron. J. Combin. 9 (2002), 10 pp.

7. T. Halverson, "Representations of the $q$-rook monoid," J. Algebra 273 (2004), 227-251.

8. T. Halverson and A. Ram, " $q$-rook monoid algebras, Hecke algebras and Schur-Weyl duality," Zap. Nauchn. Sem. St. Petersburg, Otdel. Mt. Inst. Steklov (POMI) 283 (2001), 224-250.

9. G. James and A. Kerber, "The representation theory of the symmetric group," vol. 16 of Encyclopedia of Mathematics and its Applications, Addison-Wesley Publishing Co., Reading, Mass., 1981.

10. S. König and C. Xi, "On the structure of cellular algebras," In Algebras and Modules II (Geiranger 1996) CMS Conf. Proc. 24, Amer. Math. Soc., Providence 1998, pp. 365-386.

11. S. König and C. Xi, "Cellular algebras: Inflations and Morita equivalences," J. London Math. Soc. 60(2) (1999), 700-722.

12. S. König, and C.C. Xi, "A characteristic free approach to Brauer algebras," Trans. Amer. Math. Soc. 353(4) (2001), 1489-1505.

13. W.D. Munn, "The characters of the symmetric inverse semigroup," Proc. Cambridge Philos. Soc. 53 (1957), 13-18.

14. L. Solomon, "Representations of the rook monoid," J. Algebra 256 (2002), 309-342.

15. L. Solomon, "The Iwahori algebra of $M_{n}\left(F_{q}\right)$. A presentation and a representation on tensor space," $J$. Algebra 273 (2004), 206-226.

16. C.C. Xi, "Partition algebras are cellular," Compos. Math. 119 (1999), 99-109. 